\title{
DEVELOPMENT AND CALIBRATION OF A HYSTERETIC MODEL FOR CFS STRAP BRACED STUD WALLS
}

\author{
V. Macillo, S. Shakeel, L. Fiorino * and R. Landolfo \\ Research fellow, Department of Structures for Engineering and Architecture, \\ University of Naples "Federico II", Naples, Italy \\ Research fellow, Department of Structures for Engineering and Architecture, \\ University of Naples "Federico II", Naples, Italy \\ *Assistant professor, Department of Structures for Engineering and Architecture, \\ University of Naples "Federico II", Naples, Italy \\ Full Professor, Department of Structures for Engineering and Architecture, \\ University of Naples "Federico II", Naples, Italy \\ *(Corresponding author: E-mail: Ifiorino@unina.it)
}

Received: 10 June 2016; Revised: 26 June 2016; Accepted: 13 July 2017

\begin{abstract}
Cold-formed steel (CFS) members in buildings are in use for decades, but their earthquake performance remained unexplored, until recently. Eurocodes, which provide common approach for the design of buildings in Europe still lack the information on seismic design of CFS structures. However, for the last few years, University of Naples "Federico II" CFS research group is dedicated in investigating their seismic performance. One of the focus areas is characterizing seismic behaviour of CFS strap braced stud walls. This paper presents a generalized approach adopted in simulating hysteretic behaviour of these walls under cyclic load in OpenSees software. Numerical models are developed and calibrated on the basis of available experimental results.
\end{abstract}

Keywords: Cold-formed steel, Strap braced stud walls, Hysteretic behaviour, OpenSees, Non-linear model, backbone curve

DOI: $10.18057 /$ IJASC.2018.14.3.2

\section{INTRODUCTION}

In recent years, there has been rapid increase in use of cold formed steel (CFS) for low-rise buildings in North America, Europe and Oceania. Their ability to guarantee good structural response under an earthquake has brought them in spotlight for many researchers. CFS is a common term used for products made by rolling or pressing steel at relatively low temperatures (cold working). CFS products, characterized by high structural performance, light weightiness and rapid construction presents a good alternative to traditional construction materials. There are mainly three construction techniques for CFS structures used nowadays; stick built, penalized and modular constructions. In case of stick built construction, the structure is assembled on site using the modest number of members (e.g. studs, joists and tracks) and sheathing panels. Panelized constructions are made of two-dimensional prefabricated block elements (wall and floor sub-frames with roof trusses). Modular structures use pre-engineered units with all frame elements and finishes assembled in workshop and added in horizontal or vertical direction on site.

To resist the horizontal loads, namely earthquake and wind loads, two different energy dissipative systems are used in CFS constructions, all-steel and sheathed-braced. In case of all-steel solutions, lateral resistance is usually provided by thin steel straps acting as braces in an X configuration. While for sheathing-braced solutions, steel sheets or wood based panels generally provide the lateral bracing effect to withstand horizontal loads. Currently, North American Standard AISI S400 [1] is the key reference document for seismic design of these systems. However, in recent years, 
extensive research $[2,3,4,5,6,7]$ has been carried out at University of Naples "Federico II" in order to better understand seismic performance of these CFS lateral force resisting systems and to incorporate guidelines for their seismic design in European and Italian Standards.

Iuorio et al. [7] tested three configurations (twelve tests) of CFS strap braced stud walls designed according to elastic (non-dissipative) and dissipative design approaches. Walls lateral response was investigated by carrying out tests under monotonic and cyclic loading. Additionally, they performed several tests on steel materials, elementary connections and joints between strap and gusset plate in order to study the component influence on global wall behaviour.

Macillo et al. [6] validated capacity design procedure for CFS strap braced stud walls according to European Standards. Moreover, seismic peculiarities of CFS strap braced stud walls were studied in contrast with seismic provisions for traditionally braced steel structures in European Standard EN 1998-1 [8]. Fiorino et al. [9] validated the design criteria of CFS strap braced stud wall against the provision in North American Standard AISI S213 [10] and seismic provisions for traditionally braced steel structures in European Standard EN 1998-1 [8]. Besides, they also evaluated behaviour factor at the component level based on tests performed by Iuorio et al. [7]. However, for more accurate estimation of behaviour factor, non-linear dynamic analysis and shake table test of 3D structures are needed.

In this perspective, the present paper provides the outcomes of numerical modelling of CFS strap braced stud wall in OpenSees software [11]. In particular, the calibration of pinched hysteretic material known as pinching4 [12] used to predict behaviour of CFS strap braced stud walls under dynamic loading is explained. The paper is organized as follows: Section 2 provides recent research in modelling of CFS shear walls and details about the theoretical design procedure for seismic design of CFS strap braced stud wall; Section 3 summarizes the design of walls modelled in this study, provides the description of numerical models developed for them and the calibration of pinching 4 material; Section 4 checks the reliability of developed numerical models against the test results; Sections 5 explores the adequacy of the calibrated models by using theoretical procedure for the definition of wall response; and section 6 checks their ability to simulate brittle failure modes.

\section{BACKGROUND}

\subsection{Previous Research on Numerical Modelling of CFS Shear Walls}

In the past, several researchers developed numerical models of CFS shear walls for both all steel and sheathing braced solutions. These models ranged from simple analytical to very complex finite element representations. This section summarizes the main intent behind the development of these models and their main features.

Pastor and Rodríguez-Ferran [13] presented a differential model of hysteretic behaviour for CFS strap braced walls, which can capture the slackness due to buckling of braces as well as strength and stiffness degradation. However, their model assumes that wall is able to maintain its load carrying capacity under excessive lateral displacements without ever reaching to failure.

Kim et al. [14] developed the analytical models for CFS strap braced stud walls in DRAIN-2DX [15] program. In their model, they used beam column element with plastic hinges lumped at both end for the studs. Addition of plastic hinges at the end of studs was due to the fact that they had fixed connections in earlier tested specimen of walls [16] and it accounted for additional bending stiffness of studs. For straps, they used inelastic truss bar elements. However, original hysteretic 
model of this element in DRAIN-2DX [15] did not capture severe pinched behaviour of CFS strap braced stud walls, therefore they added a gap property to truss element to capture the complete behaviour.

Comeau et al. [17] evaluated the seismic performance factors for CFS strap braced stud walls using Incremental Dynamic Analysis (IDA) based on FEMA P695 [18] methodology. Bilinear spring elements with strain hardening and slackness characteristics were used to model hysteretic response on walls in Ruaumoko [19] software. Mirzaei et al. [20] developed an elastic analytical model with fixed connections except for the end of braces, in order to study the effect of wall aspect ratio on the moment generated in chord studs due to frame action.

In addition to above mentioned analytical models, several finite element models were also developed. Gad et al. [21] developed FE models in ANSYS [22] for Plasterboard lined CFS frame without bracing in order to study effect of presence of different boundary conditions and aspect ratio on ultimate capacity of wall using nonlinear static analysis. Later on, they combined these models with models of braced frame developed by Barton [23]. In the combined model, straps were modelled as series of three nonlinear springs: strap-frame connection, solid strap and strap tensioner unit system.

Zeynalian and Ronagh [24] developed finite element models of CFS strap braced stud walls with additional brackets at corner of walls. A nonlinear static analysis was conducted in ANSYS [22] with varying the length of brackets for each model in order to optimize their seismic response.

Shamim et al [25-26] performed several shake table tests on CFS shear walls braced with wood based panels and steel sheets in order to check their behaviour under an earthquake and to propose seismic design provisions for North American standards. They developed non-linear model of walls in OpenSees [11], which were calibrated based on their experimental results. Then, these non-linear model of walls were used in 3d Incremental Dynamic Analysis (IDA) of archetype buildings to evaluate the seismic performance factors according to FEMA P695 [18]. In sheathed braced walls, sheeting material is responsible for energy dissipation. Shamim et al. modelled sheets by equivalent diagonals as truss elements (braces). Pinching4 material [12] was used to represent the non-linear behaviour of walls. Additionally, they used Zero length springs to model tension anchors at wall corners. For wall frame profiles, they used Elastic beam column elements. P-delta effects were modelled using an additional frame in direction of seismic loads using rigid Truss and Beam column elements having Co-rotational coordinate transformation.

In USA, major research of modelling of CFS sheathed braced shear walls, ranging from hysteretic characterization of single walls to complete 3D model development, is carried under CFS NEES project. Leng et al. [27] modelled a complete double storey CFS building and performed non-linear Incremental Dynamic Analysis (IDA) using OpenSees [11]. Equivalent energy elastic plastic (EEEP) and Pinching4 material models were fitted to the tested data by Liu et al. [28]. It was shown that the EEEP models are only appropriate for static pushover analysis and Pinching 4 material [12] models are capable of reasonably capturing the full hysteretic response, including degradation and were recommended for use in non-linear time history analysis.

Kechidi et al. [29] presented an accurate model to represent pinched hysteretic behaviour of wood and steel sheathed shear walls. Developed model were implemented in OpenSees [11] as user defined uniaxial material instead of Pinching4 material. Their models had both strength and stiffness degradation capabilities with curvature in loading and unloading branches. They used a pair of rigid truss element in X configuration with an equivalent non-linear Zero Length element in the middle having user-defined material to represent the wall behaviour in OpenSees. 
To sum up, numerical models presented in Pastor and Rodríguez-Ferran [13], Kim et al. [14] and Comeau et al. [17] lacked the ability to capture brittle failure modes for CFS strap braced stud walls. Available models developed for CFS strap braced stud walls did not represent all wall structural components, except models presented in Kim et al. [14]. In addition, finite element models presented in Gad et al. [21] and Zeynalian and Ronagh [24] only represented the static behaviour of CFS strap braced stud walls and cannot be used in analysis of complete buildings due their complexity. In this context, there is a need to develop a numerical model which can capture brittle and ductile failure modes, properly represents the contribution of all components to lateral force resistance under dynamic and cyclic loads and is computationally efficient to be used in models of complete 3D buildings.

\subsection{Procedure for Predicting the Wall Response}

CFS strap braced stud wall (Figure 1) is an all steel solution to resist horizontal loads in CFS structures. The lateral system consists of concentric X bracings, made of very slender steel straps placed on both sides of walls, which are considered active only in tension. Any lateral load applied on walls is absorbed by straps in tension transmitting significant axial compression force on studs located at the ends of the wall.

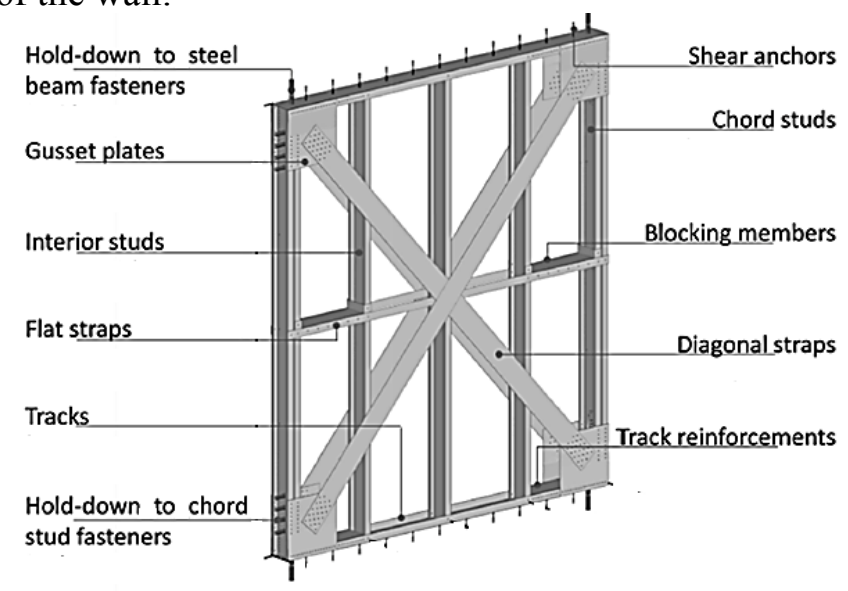

Figure 1. Typical CFS Strap Braced Stud Wall

CFS walls are made of series of studs (columns) usually placed $600 \mathrm{~mm}$ apart fitted on horizontal tracks. External studs (chord studs) can either be back to back lipped C-channel or box cross section, in order to avoid buckling due to compression force transmitted by straps. Track members are usually made of un-lipped U cross sections. Gusset plates are usually provided at corner of walls in order to attach straps with the wall frame, generally with self-drilling screws. Flat straps can be attached at the mid height of the wall in order to reduce the buckling length of studs. Local buckling of tracks can be avoided by placing channel section profiles to form a box shape. Hold-down devices and shear anchors are used to connect the wall to foundation or to other floors. Three main structural components of the wall: diagonal in tension $\left(d_{d}\right)$, tension anchors $\left(d_{a}\right)$ and diagonal to frame connections $\left(d_{c}\right)$ contribute to total top displacement $(\mathrm{d})$ of the wall, when it is subjected to a lateral load. Hence, the total lateral displacement is calculated according to Eq. 1

$d=d_{d}+d_{a}+d_{c}$

The lateral resistance of the wall subjected to in plane loading can be evaluated by taking into account strength associated with each wall component [6]. Table 1 provides the procedure for the evaluation of design strength of each wall component. For each wall component, one or more 
failure mechanism can be identified and then strength associated with the weakest failure mode define the design lateral strength of the wall. Therefore, the lateral design strength of the wall $\left(H_{c}\right)$ can be computed according to Eq. 2 :

$H_{c}=\min \left(H_{c, c}, H_{c, d}, H_{c, g}, H_{c, s}, H_{c, t}, H_{c, a}\right)$

For seismic design, current trends propose to design the seismic resisting system according to capacity design criteria, which direct to the failure mechanism that maximizes the walls ductility. In case of CFS strap braced stud walls, the most ductile failure mechanism is the yielding of steel straps in tension. Therefore, the net section rupture of straps connections as well as the failure of studs, tracks and anchors should be avoided through an appropriate over strength.

Table 1. Design Wall Strength Associated to each Wall Component

\begin{tabular}{|c|c|c|}
\hline Strength of the wall component & Failure modes & Design Formula \\
\hline $\begin{array}{l}H_{c, c^{-}} \text {wall strength associated to } \\
\text { the diagonal to strap connection }\end{array}$ & $\begin{array}{l}\text { Bearing failure in the strap of a } \\
\text { screw }-F_{b, R d} \\
\text { Shear failure of a screw- } F_{v, R d}\end{array}$ & $n_{d} \cdot n_{s} \cdot \min \left(F_{b, R d}, F_{v, R d}\right) \cos \theta$ \\
\hline $\begin{array}{l}H_{c, d} \text {-wall strength associated to } \\
\text { the yielding of the diagonal strap }\end{array}$ & $\begin{array}{l}\text { Yielding of a steel the strap- } \\
N_{p l, R d} \\
\text { Net section failure of a strap- } \\
N_{u, R d}\end{array}$ & $n_{d} \cdot \min \left(N_{p l, R d}, N_{u, R d}\right) \cos \theta$ \\
\hline $\begin{array}{l}H_{c, g^{-}} \text {wall strength associated to } \\
\text { the net section failure of the } \\
\text { gusset plate }\end{array}$ & $\begin{array}{l}\text { Net section failure of a gusset } \\
\text { plate- } F_{n, R d}\end{array}$ & $n_{d} \cdot F_{n, R d} \cos \theta$ \\
\hline $\begin{array}{l}H_{c, s}-\text { wall strength associated to } \\
\text { the buckling of the studs }\end{array}$ & Buckling of a chord stud- $N_{s, R d}$ & $\frac{N_{s, R d}-N_{E d, G}}{h} L$ \\
\hline $\begin{array}{l}H_{c, t^{-}} \text {wall strength associated to } \\
\text { the buckling of the track }\end{array}$ & Buckling of a track- $N_{t, R d}$ & $N_{t, R d}$ \\
\hline $\begin{array}{l}H_{c, a^{-}} \text {wall strength associated to } \\
\text { the tension and shear anchors }\end{array}$ & $\begin{array}{l}\text { Strength of a tension anchor- } \\
N_{a, R d} \\
\text { Strength of a shear anchor- } \\
V_{a, R d}\end{array}$ & $\min \left(\frac{N_{a, R d}+N_{E d . G}}{h} L ; n_{a} V_{a, R d}\right)$ \\
\hline \multicolumn{3}{|c|}{$\begin{array}{l}\boldsymbol{n}_{\boldsymbol{d}} \text { is the total number of straps in tension; } \boldsymbol{n}_{\boldsymbol{s}} \text { is the total number of screws in a strap-to-frame connection; } \boldsymbol{N}_{\boldsymbol{E} \boldsymbol{d}, \boldsymbol{G}} \text { is the } \\
\text { axial force due to gravity loads; } \boldsymbol{\theta} \text { is the angle of strap with respect to horizontal; } \boldsymbol{L} \text { is the length of the wall; } \boldsymbol{H} \text { is the height } \\
\text { of the wall }\end{array}$} \\
\hline
\end{tabular}

\section{Numerical Model Development}

\subsection{Design of Walls}

In this study, two walls configurations are selected in order to calibrate the proposed model. These walls are designed following the dissipative design approach summarized in section 2.2 and described in detail by Macillo et al. [6]. The selected walls correspond to light (WLD) and heavy (WHD) dissipative capacity walls tested by Iuorio et al. [7]. Therefore, for the sake of simplicity, numerical model of walls presented in this study are also named as WLD and WHD. The cyclic test results are used for calibrating the numerical models for the walls. In particular, two identical set of experiments ( $\mathrm{C} 1$ and $\mathrm{C} 2)$ are available for both WLD and WHD wall configurations.

Table 2 summarizes the general design characteristics of both wall configurations, while Table 3 gives the design and nominal values of lateral wall strength associated to each wall component. 
Table 2. Design of CFS Strap Braced Stud Walls

\begin{tabular}{|c|c|c|}
\hline & WLD & WHD \\
\hline Width x Height & $2400 \times 2700 \mathrm{~mm}$ & $2400 \times 2700 \mathrm{~mm}$ \\
\hline \multirow{2}{*}{ Chords studs } & Back to back channel section (S350) & Back to back channel section (S350) \\
\hline & $\mathrm{C} 150 \times 50 \times 20 \times 1.5 \mathrm{~mm}^{\mathrm{a}}$ & $\mathrm{C} 150 \times 50 \times 20 \times 3 \mathrm{~mm}^{\mathrm{a}}$ \\
\hline Straps & $70 \times 2 m^{b}(\mathrm{~S} 235)$ & $140 \times 2 \mathrm{~mm}^{\mathrm{b}}(\mathrm{S} 235)$ \\
\hline Diagonal to frame connection & No. $154.8 \times 16 \mathrm{~mm} \mathrm{screws}^{\mathrm{c}}$ & No.25 $6.340 \mathrm{~mm} \mathrm{screws}^{\mathrm{d}}$ \\
\hline Design wall strength & $42 \mathrm{kN}$ & $85 \mathrm{kN}$ \\
\hline Theoretical wall strength ${ }^{\mathbf{e}}$ & $56 \mathrm{kN}$ & $112 \mathrm{kN}$ \\
\hline Tension anchors & M24 class 8.8 bolt rods & M24 class 8.8 bolt rods \\
\hline Shear anchors & $\begin{array}{c}\text { M8 class } 8.8 \text { bolts spaced at } 300 \mathrm{~mm} \text { on } \\
\text { center }\end{array}$ & $\begin{array}{c}\text { M8 class } 8.8 \text { bolts spaced at } 300 \mathrm{~mm} \\
\text { on center }\end{array}$ \\
\hline $\begin{array}{l}\text { Hold down to chord stud } \\
\text { fastener }\end{array}$ & 4 M16 class 8.8 bolts & $4 \mathrm{M} 16$ class 8.8 bolts \\
\hline Theoretical wall stiffness & $4.37 \mathrm{kN} / \mathrm{mm}$ & $7.52 \mathrm{kN} / \mathrm{mm}$ \\
\hline $\begin{array}{l}\text { a. C-section: outside-to-outsic } \\
b . \quad \text { Width } \mathrm{x} \text { thickness. } \\
\text { c. Nominal diameter } \mathrm{x} \text { length, } \\
\text { d. Nominal diameter } \mathrm{x} \text { length, } \\
\text { e. Theoretical wall strengths } p\end{array}$ & $\begin{array}{l}\text { web depth } \mathrm{x} \text { outside-to-outside flange size } \\
\text { odified truss head self-drilling screws. } \\
\text { xagonal flat washer head self-drilling scre } \\
\text { dicted on the basis of average experimentc }\end{array}$ & utside-to-outside lip size $\mathrm{x}$ thickness. \\
\hline
\end{tabular}

\subsection{Model Description}

2D models with 3 degrees of freedoms, i.e. vertical and horizontal translation and rotation in plane are developed in OpenSees [11] for both selected walls. Figure 2 shows generic OpenSees model developed for CFS strap braced stud walls.

Chord Studs are modelled using truss elements with a Uniaxial elastic material, having modulus of elasticity of $210 \mathrm{GPa}$. The OpenSees MinMax material is used in conjunction with uniaxial elastic material in order to simulate the chord stud failure due to tension or global buckling. OpenSees MinMax material is always used in conjunction with another material, which define its stress-strain behaviour, and it enforces the threshold values of strain, beyond which strength and stiffness of element are set to zero. In the specific case, threshold values which govern the occurrence of failure of studs in tension $\left(\varepsilon_{\mathrm{t}, \mathrm{Rk}}\right)$ and compression $\left(\varepsilon_{\mathrm{b}, \mathrm{Rk}}\right)$ are calculated using Eqs. 3 and 4.

$$
\begin{gathered}
\varepsilon_{t, R k}=\frac{N_{t, R k}}{A \cdot E} \\
\varepsilon_{b, R k}=\frac{N_{b, R k}}{A \cdot E}
\end{gathered}
$$

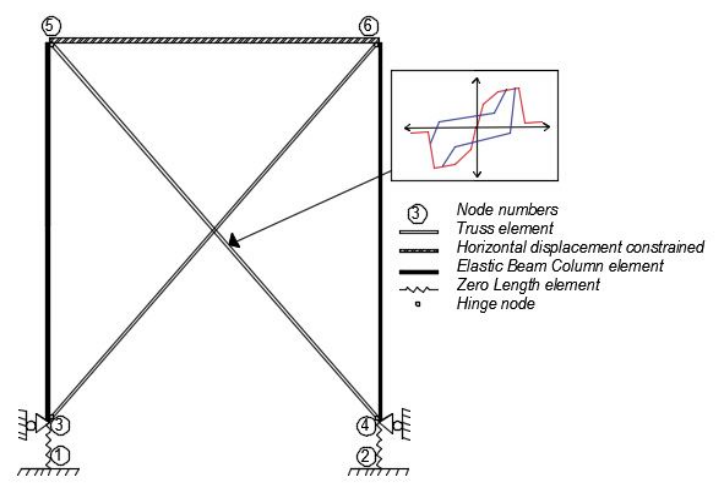

Figure 2. OpenSees Model for CFS Strap Braced Stud Wall 
where, $N_{t, R k}$ and $N_{b, R k}$ are nominal resistances corresponding to the tensile and buckling failure of chord studs, respectively, $\mathrm{E}$ is the Young's modulus of steel and $\mathrm{A}$ is gross cross section area. $N_{t, R k}$ and $N_{b, R k}$ are obtained according to prescriptions given in EN 1993-1-1 [30] and EN 1993-1-3 [31]. Straps are modelled with Truss elements using Pinching 4 material. Information about the calibration of strap's pinching4 material is provided in section 3.3. Stiffness contribution through hold down anchors to foundation is integrated by using Zerolength elements of unit area at bottom of stud's end. ElasticMultiLinear material with a stiffness of $30 \mathrm{kN} / \mathrm{mm}$ in tension, defined on the basis of experimental results of Iuorio et al. [7] and a very high stiffness $(10,000 \mathrm{KN} / \mathrm{mm})$ in compression is used for Zerolength elements. In OpenSees definition of ElasticMultiLinear material, the nonlinear stress-strain relationship is given by a multi-linear curve that is defined by a set of points. The material behaviour is nonlinear but elastic without any energy dissipation. MinMax material is used in conjunction with ElasticMultiLinear material in order to take into account tensile failure of anchors. Tensile strain in MinMax material for anchors is set to not exceed the nominal tensile strain $\varepsilon_{\mathrm{a}, \mathrm{Rk}}$ of anchors preventing any tensile rupture in them while there is no strain limit in compression. Nominal tensile strain in anchors $\varepsilon_{\mathrm{a}, \mathrm{Rk}}$ is calculated using Eq. 5 :

$\varepsilon_{a, R k}=\frac{N_{a, R k}}{k_{a}}$

where $N_{a, R k}$ is the nominal tensile resistance of anchors and $\mathrm{k}_{\mathrm{a}}$ is the stiffness of tension anchor equal to $30 \mathrm{kN} / \mathrm{mm}$. Rigid diaphragm effect is incorporated by constraining the horizontal displacements at the wall top, using EqualDOF command between nodes 5 and 6 . P- $\Delta$ effects are neglected in OpenSees model because there were no gravity loads present in the test [7].

In order to check consistency of the developed model against test results [9], non-linear quasi static analysis using cyclic load is performed. Same cyclic protocol as used in the reference tests [7] is imposed at top of walls. In experimental activity, CUREE ordinary ground motions reversed cyclic load developed by Krawinkler et al. [21] for wood walls and modified by Velchev et al. [22] for CFS strap braced walls was used. The loading protocol consists of initiation, primary and trailing cycles (Figure 3). First six cycles of the protocol are initiation cycles, which serve to check loading equipment, measuring devices and force deformation response at small amplitudes. After execution of initiations cycles, there are series of primary and trailing cycles. Primary cycles have bigger amplitude than all of the preceding cycles and is followed by small amplitude cycles, which are called as trailing cycles. All trailing cycles have amplitude equal to $75 \%$ of the amplitude of the preceding primary cycle. In this paper, primary cycles before the cycle number 32 are identified as minor primary cycles, while after 32 they are recognized as major primary cycles.

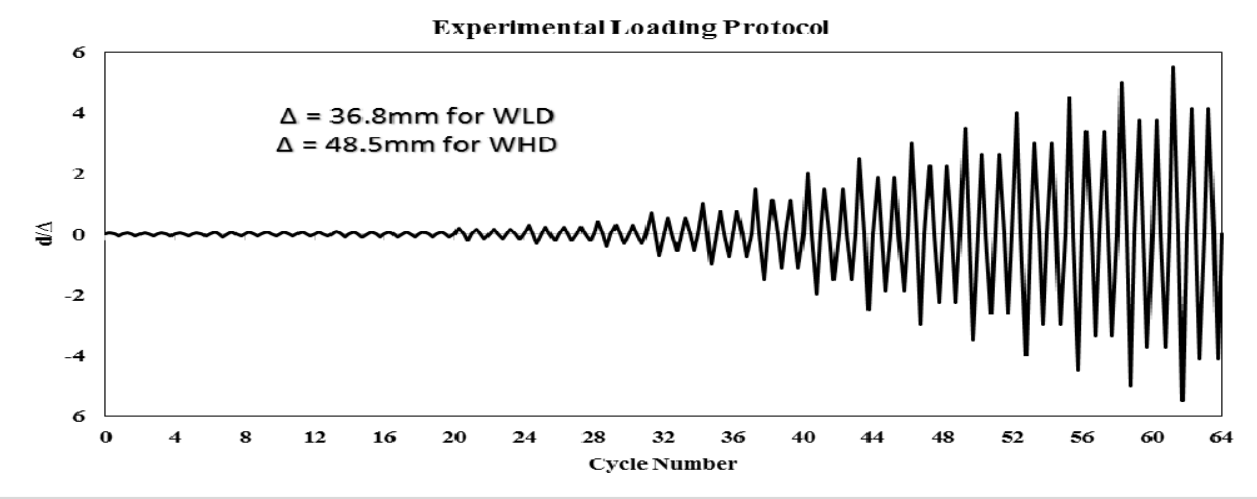

Figure 3. Experimental Cyclic Loading Protocol [6] 


\subsection{Hysteretic Characterization}

Pinching4 material [12] is used to represent pinched hysteretic response of straps. Pinching4 material is a uniaxial material that can represent pinched load deformation response with the ability to exhibit degradation under cyclic loading. OpenSees [11] definition of Pinching 4 material requires a set of 39 parameters to be defined in order to represent the non-linear hysteretic behaviour. It includes 16 parameters for the definition of the backbone curve $(e P f 1, e P d 1, e P f 2$, ePd2, ePf3, ePd3, ePf4, ePd4, eNf1, eNd1, eNf2, eNd2, eNf3, eNd3, eNf4, eNd4), 6 parameters for defining the cyclic behaviour ( $r D i s p P$, rDispN, rForceP, rForceN, uForceP and uForceN), 5 parameters for governing the strength degradation $(g F 1, g F 2, g F 3, g F 4, g F L i m), 5$ parameters for controlling the unloading stiffness degradation $(g K 1, g K 2, g K 3, g K 4, g K L i m), 5$ parameters for controlling the reloading stiffness degradation $(g D 1, g D 2, g D 3, g D 4, g D L i m)$ and 2 parameters for limiting the maximum degradation in each cycle ( $g E, d m g T y p e)$. The key parameters used in this study to define Pinching4 material are illustrated in Figure 4. These parameters are selected based on available experimental results [7]. Out of the 39 parameters required for definition of Pinching 4 material, only 22 parameters are calibrated to represent the wall behaviour in this study. The calibration process of these parameters is explained in following passages. The rest of 17 parameters representing the strength and stiffness degradation in hysteretic behaviour are taken as 0 , because this type of degradation behaviour was not observed during the experiment and is not significant for the investigated system.

In particular, the material law is defined by a 4-points multi-linear backbone envelope in both positive and negative directions, which is calibrated on the basis of experimental results. In Figure 4, points (ePd1, ePf1) to (ePd4, ePf4) and (eNd1, eNf1) to (eNd4, eNf4) represent the positive and negative branch of the backbone envelope curve in terms of stress and strain, respectively. This 4-points backbone curve is adjusted to capture entire envelope of hysteretic behaviour. First and second point of the backbone curve lies within elastic limit, third point represents the wall strength and fourth point is used to capture the post peak behaviour. Theoretically, there should be no energy dissipation until wall is in linear state, but experimental results showed non-linear behaviour from start. In order to catch this type of behaviour, the first point of the backbone curve is positioned at $50 \%$ strength of the second point where the second point corresponds to the elastic limit. The third point is the maximum strength achieved in experimental results. Experimental results also showed a vertical drop of strength after the peak. This decreasing of strength may lead to convergence problems in numerical analysis. Therefore, last branch of the backbone curve is aligned with little positive slope downwards using fourth point on backbone.

Eqs. 6 to 8 are used to transform force and displacement from experimental results into stress and strain of Pinching 4 material used in the numerical model.

$$
f=\frac{f}{\cos (\theta)}
$$

$\delta=\Delta \cos (\theta)$

strain $=\frac{\delta}{\mathrm{i}}=\frac{\Delta \cos (\theta)}{\mathrm{i}}$

where, $F$ is the force applied during the test, $\Delta$ is the top wall displacement recorded during the test, $f$ is the stress in a strap of unit area, $\delta$ is the axial deformation in the strap, $l$ is the length of the strap and $\theta$ is the angle of the diagonal strap respect to bottom track. 


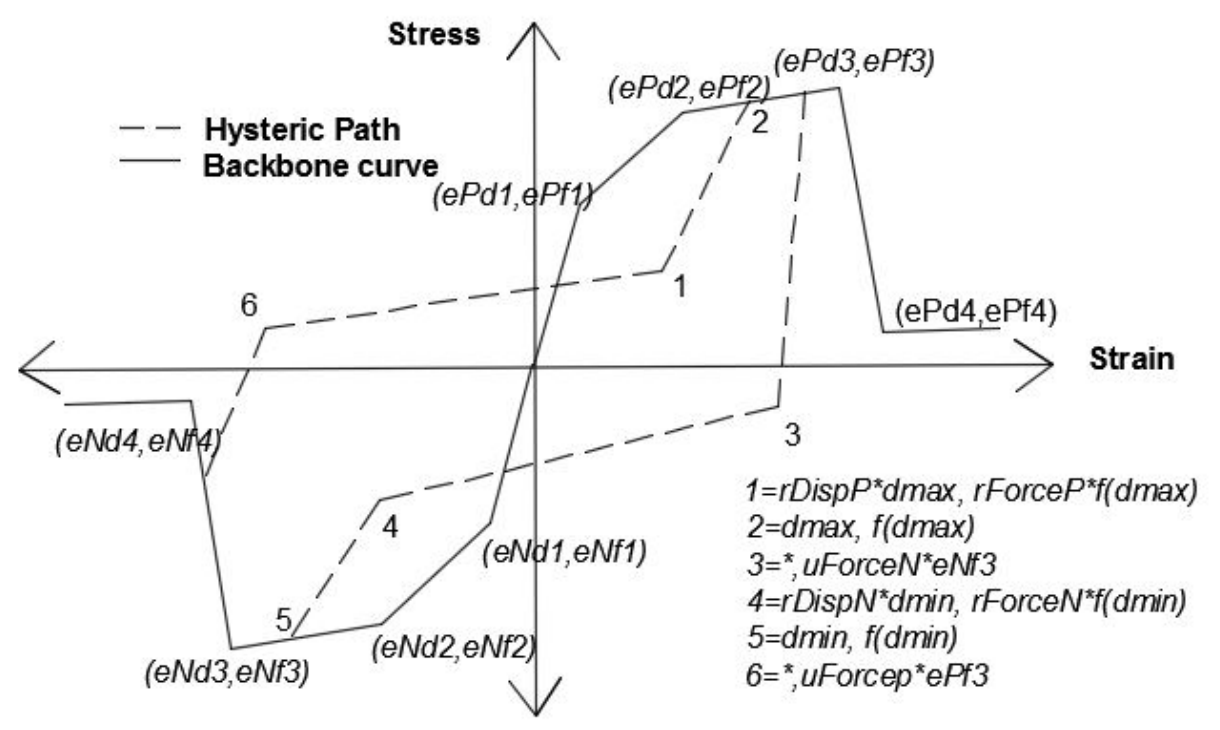

Figure 4. OpenSees Definition of Pinching4 Material

Unloading and reloading paths are controlled by series of force and displacement ratios, which include positive and negative $u$ Force, $r$ Disp and rForce parameters. As regards positive branches, $u F o r c e P$ defines the ratio between the strength developed upon unloading and maximum strength of the positive backbone curve. $r$ DispP and $r F o r c e P$ mark the strength and displacement at which reloading occurs. In particular, $r F o r c e P$ is the ratio between the strength of reloading point and strength at maximum positive displacement of preceding cycles. $r D i s p P$ is the ratio between the displacement of the reloading point and maximum positive displacement of preceding cycles. Obviously, same definitions apply for negative branches (uForceN, rDispN, rForceN).

For proposed models, symmetric values of all parameters are used for both negative and positive branches of hysteretic path. In particular, uForce, $r$ Disp and $r$ Force are selected and calibrated in a way to achieve good match with respect to experimental results, in terms of force vs. displacement relationship and dissipated energy. Since in experimental results unloading branch nearly approaches zero for all cycles, therefore uForce is taken equal to 0 . The reloading points on experimental curves are characterized by very low values of force, therefore $r F o r c e$ is set equal to 0.01 ( $r$ Force needs to be more than 0 ). Conversely, $r$ Disp is calibrated by varying the values in the range from 0.60 to 0.90 and selecting the value that minimizes, for each test, the square error in terms of dissipated energy. The obtained values for rDisp are 0.80 and 0.70 for WLD and WHD wall configurations, respectively. Table 4 summarizes the properties of Pinching 4 material used for WLD and WHD wall configurations. As stated earlier that two identical sets of experimental results $\mathrm{C} 1$ and $\mathrm{C} 2$ are available for both WLD and WHD wall configurations from the cyclic tests performed by Iuorio et al. [7], therefore a numerical model is developed for each set.

\section{COMPARISON BETWEEN NUMERICAL MODELS AND EXPERIMENTAL RESULTS}

Numerical results are compared with experimental ones both in terms of force vs. displacement response curves and energy dissipation. Numerical forces and displacements of walls are measured by a set of recorders in OpenSees and post processed with MATLAB subroutine to plot different parameters. 
Figures 5 and 6 illustrate force vs displacement response curves of both numerical models and experimental results along with the comparison of typical small and large amplitude cycles for WLD-C1 and WLD-C2 walls. It can be seen that numerical models are able to capture experimental hysteretic response in terms of overall shape and location of peaks. Adjustment of the first point of the backbone curve in mid of second point allowed the numerical models to capture the non-linear response observed from beginning of experimental response.

Figure 7 and 8 illustrate force vs displacement response curves of both numerical models and experimental results along with the comparison of typical small and large amplitude cycles for the WHD-C1 and WHD-C2 walls. Also, in this case numerical models are able to capture experimental hysteretic response in terms of overall shape and location of peaks with minute differences. It has to be noticed that, in the test of WHD-C2 wall the net section failure occurred in one straps at cycle no. 40. This unpredicted phenomenon resulted in strength reduction of about $45 \%$ in negative phase of cyclic load for displacement more than $75 \mathrm{~mm}$. Obviously, this brittle rupture cannot be accounted for by the numerical model.

Quantitative comparison of the energy dissipation of numerical and experimental results is made using Eqs. 6 to 9.

$$
\begin{aligned}
& C E_{e, j}=\sum_{i=1}^{j} E_{e, i},[i, j \leq n] \\
& C E_{n, j}=\sum_{i=1}^{j} E_{n, i},[i, j \leq n] \\
& \Delta_{E, i}=\frac{E_{n, i}-E_{e, i}}{E_{e, i}} \times 100,[i \leq n] \\
& \Delta_{C E, j}=\frac{C E_{n, j}-C E_{e, j}}{C E_{e, j}} \times 100,[j \leq n]
\end{aligned}
$$

Where $C E_{e, j}$ and $C E_{n, j}$ represent the cumulative energy dissipated for $j^{\text {th }}$ cycle of the loading protocol obtained accounting for numerical and experimental results, respectively; $E_{n, i}$ and $E_{e, i}$ represent the energy dissipated in $i^{\text {th }}$ cycle of numerical and experimental results, respectively; $\Delta_{\mathrm{E}, \mathrm{i}}$ is the percentage difference of the energy dissipation for $i^{\text {th }}$ cycle of loading protocol between numerical and experimental results; $\Delta_{\mathrm{CE}, \mathrm{j}}$ is the percentage difference of the cumulative energy for the $j^{\text {th }}$ cycle of loading protocol between numerical and experimental results; $n$ is the last cycle of the loading protocol.

Figures 9 and 10 show the comparison in terms of cumulative and cycle by cycle energy dissipation between the numerical and experimental results for the WLD-C1 and WLD-C2 walls, respectively. In case of trailing cycles of the loading protocol, the numerical model captures the energy dissipation quite well. For major primary cycles, the average of the absolute values of $\Delta_{\mathrm{E}, \mathrm{i}}$ is quite small and results $7.0 \%$ and $3.8 \%$ for WLD-C1 and WLD-C2 walls, respectively. A similar result is observed in terms of cumulative energy, with the average of the absolute values of the $\Delta_{\mathrm{CE}, \mathrm{j}}$ calculated for cycles from 32 to $n$ equal to $8.9 \%$ and $7.6 \%$ for WLD-C1 and WLD-C2 walls, respectively; whereas the error $\Delta_{\mathrm{CE}, \mathrm{n}}$ (at the $n^{\text {th }}$ cycle) is about $2 \%$ for both walls. 

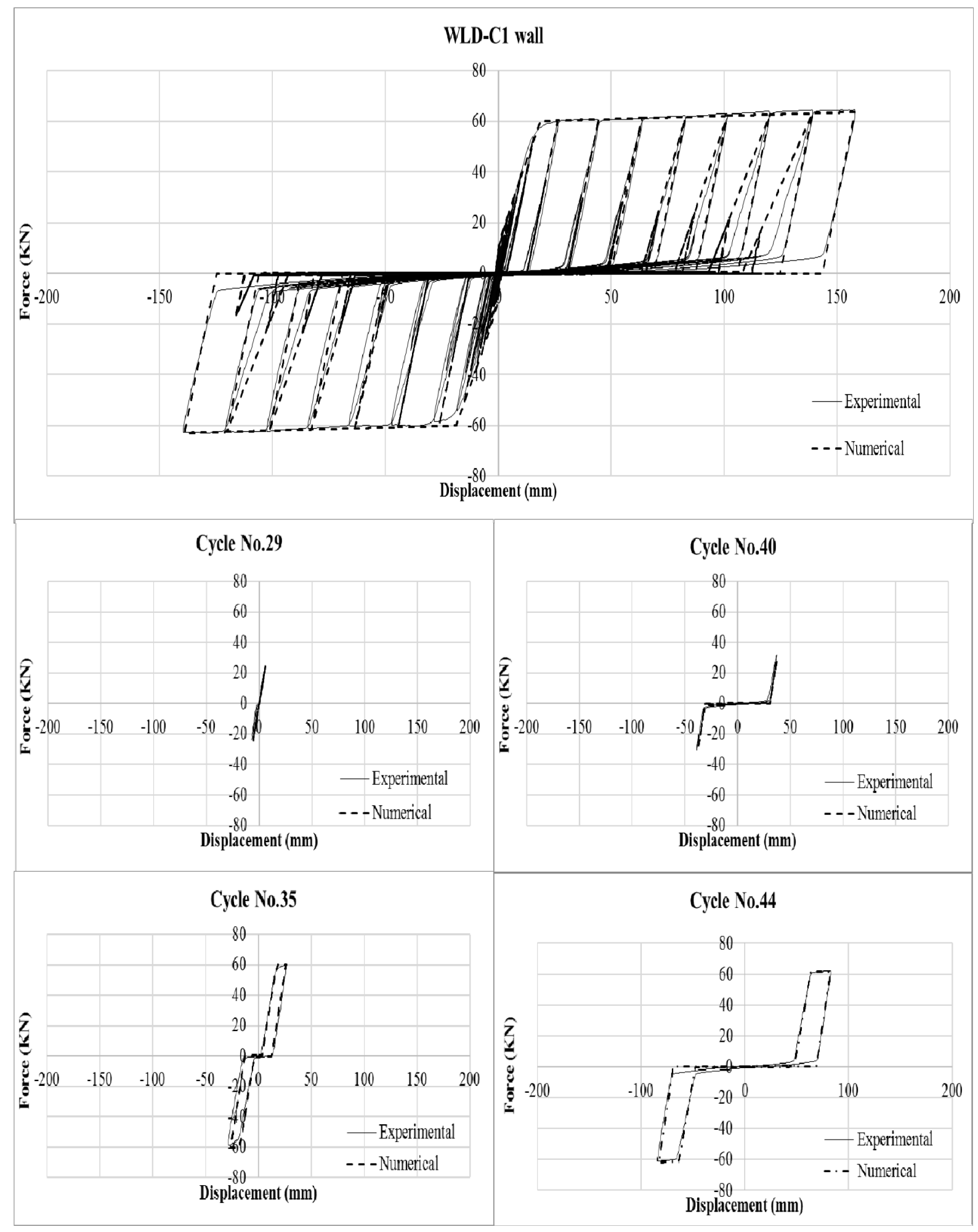

Figure 5. Comparison of Cyclic Force vs Displacement Diagrams of Numerical Models and Experimental Results for the WLD-C1 Wall 

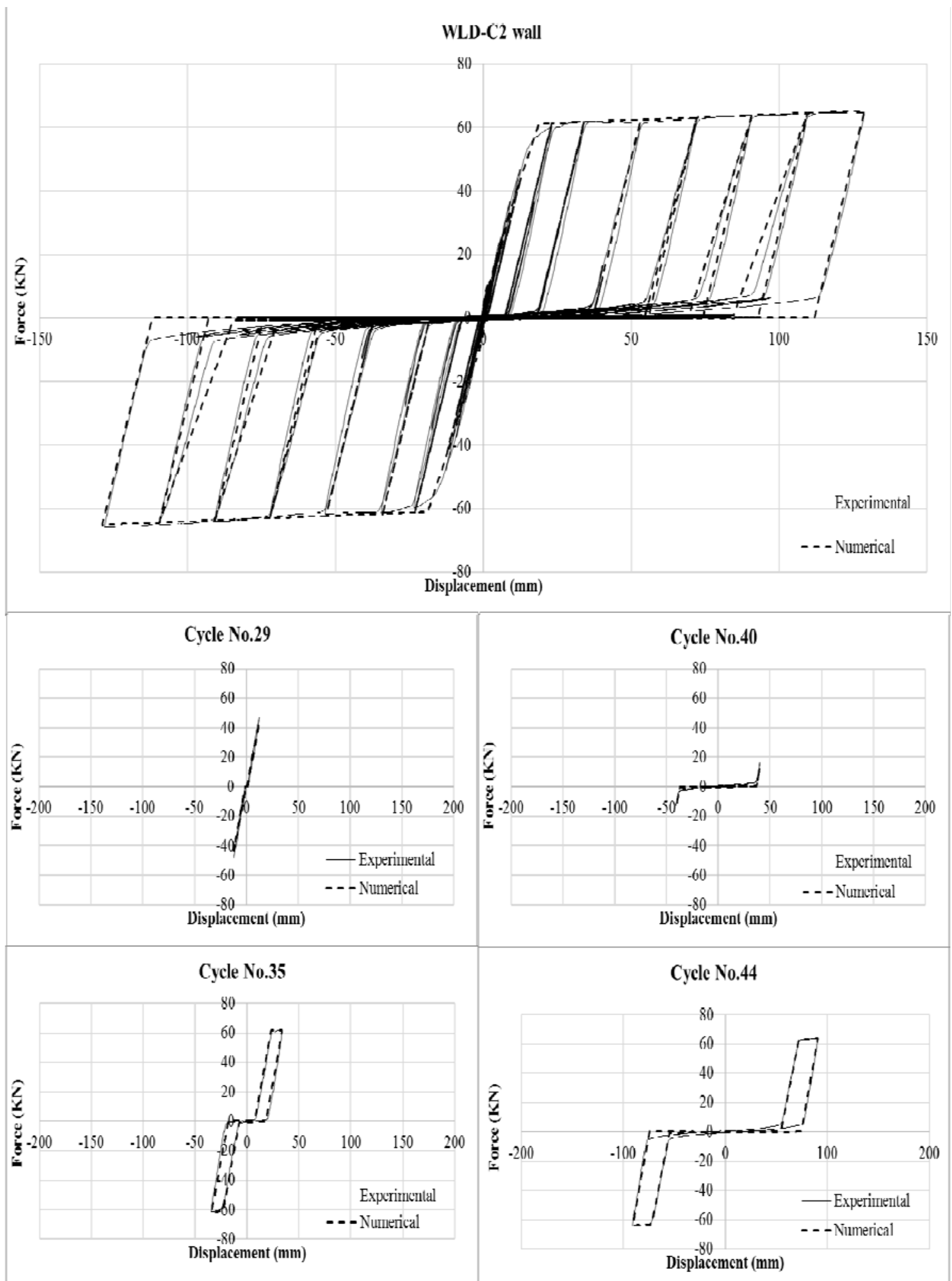

Figure 6. Comparison of Cyclic Force vs Displacement Diagrams of Numerical Models and Experimental Results of WLD-C2 for Wall 

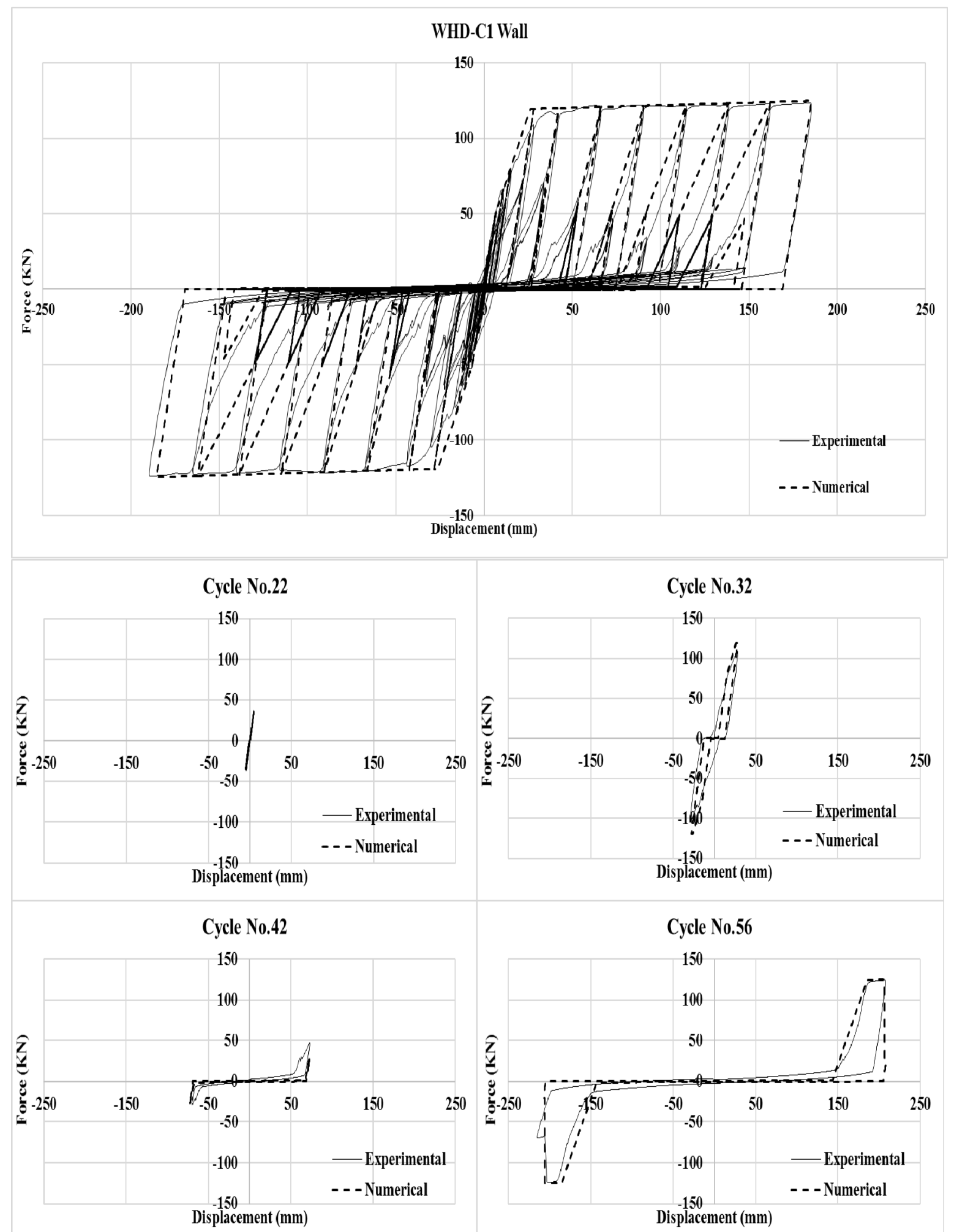

Figure 7. Comparison of Cyclic Force vs Displacement Diagrams of Numerical Models and Experimental Results for WHD-C1 Wall 


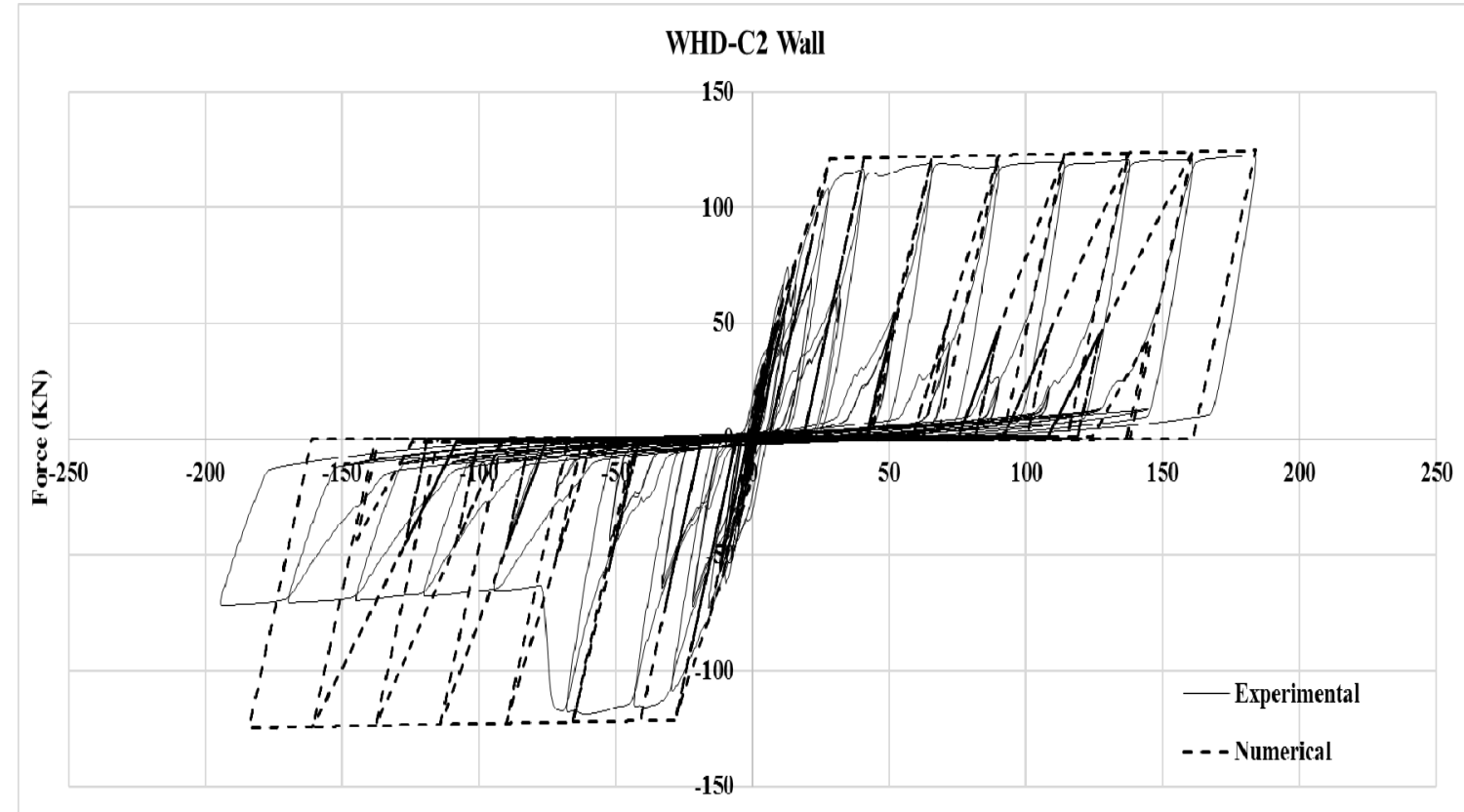

Displacement (mm)

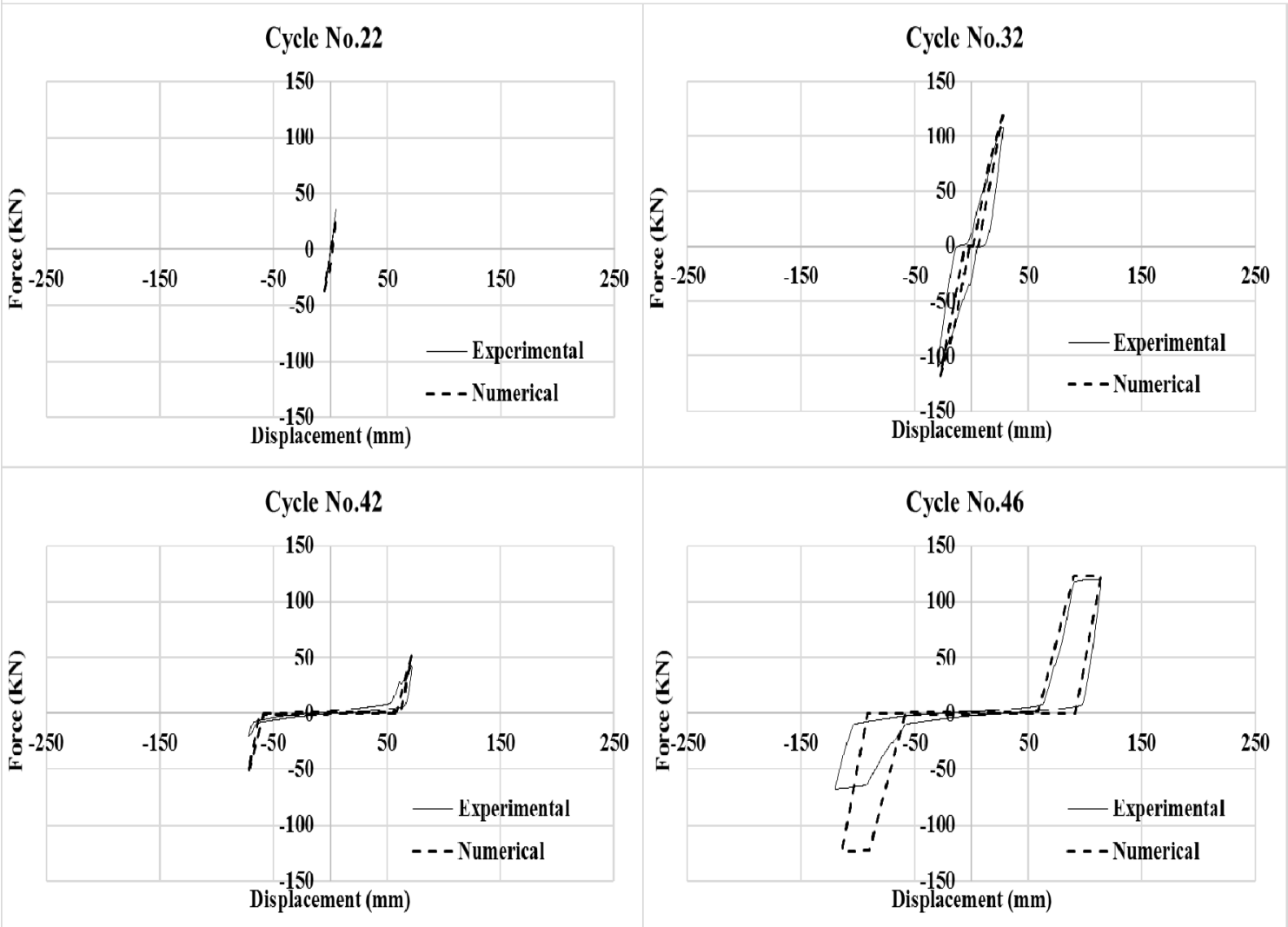

Figure 8. Comparison of Cyclic Force vs Displacement Diagrams of Numerical Models and Experimental Results for WHD-C2 Wall 


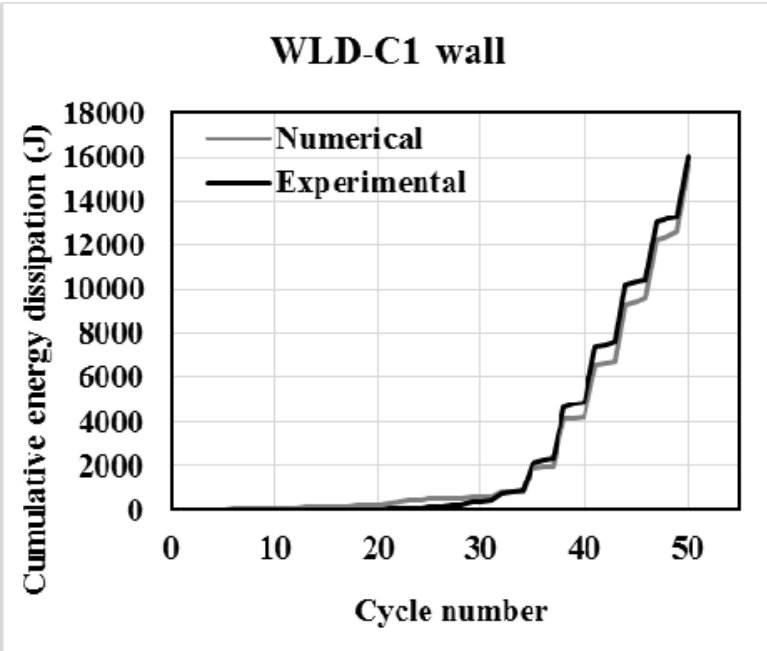

(a)

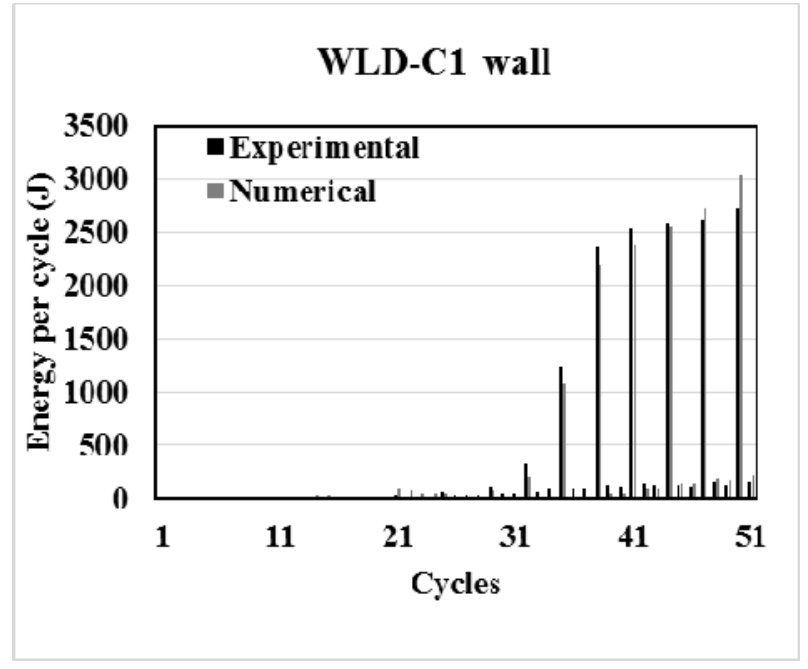

(b)

Figure 9. Comparison of a) Cumulative Energy Dissipation of Experimental and Numerical Results for the WLD-C1 Wall b) Cycle by Cycle Energy Dissipation of Experimental and Numerical Results for the WLD-C1 Wall

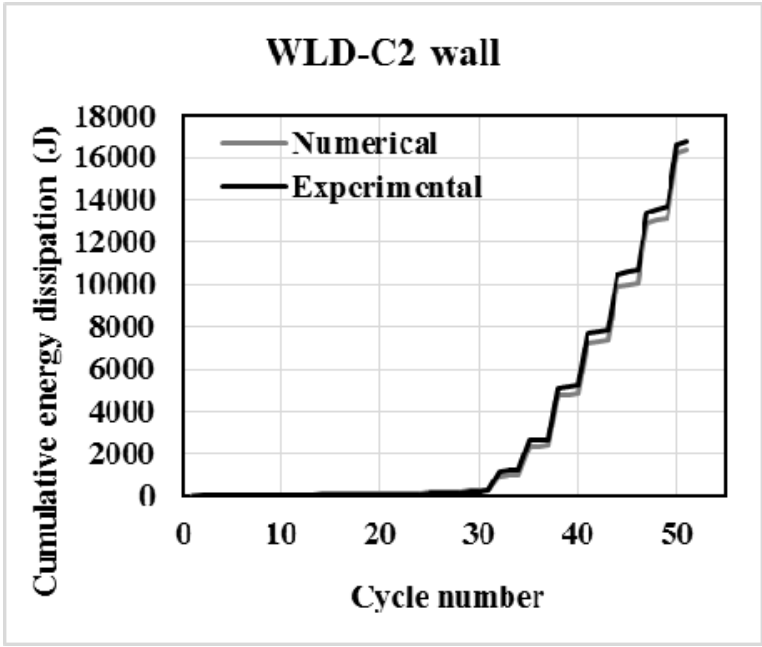

(a)

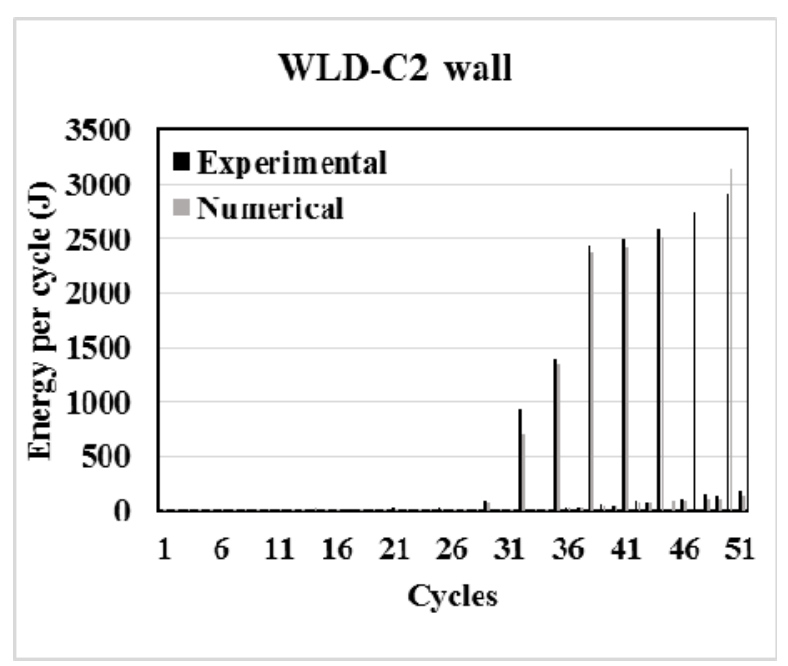

(b)

Figure 10. Comparison of a) Cumulative Energy Dissipation of Experimental and Numerical Results for the WLD-C2 Wall b) Cycle by Cycle Energy Dissipation of Experimental and Numerical Results for the WLD-C2 Wall 


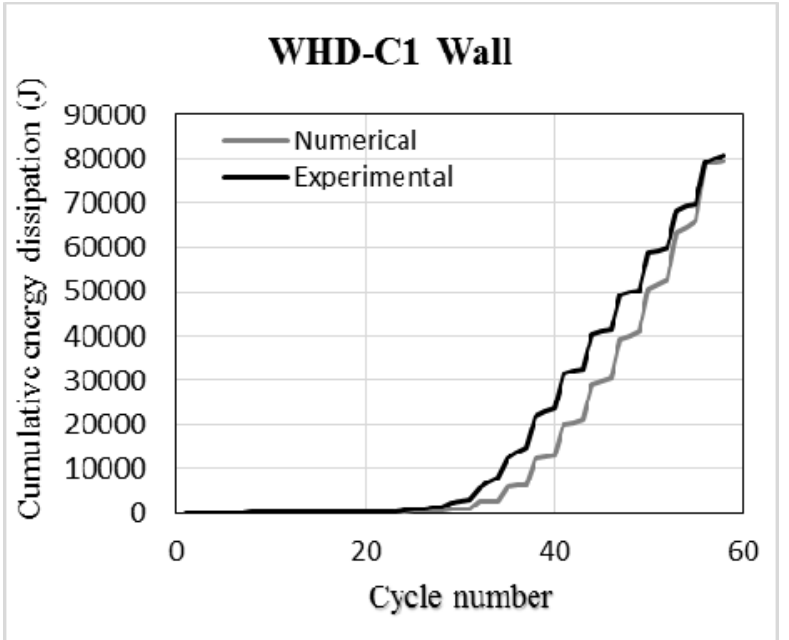

(a)

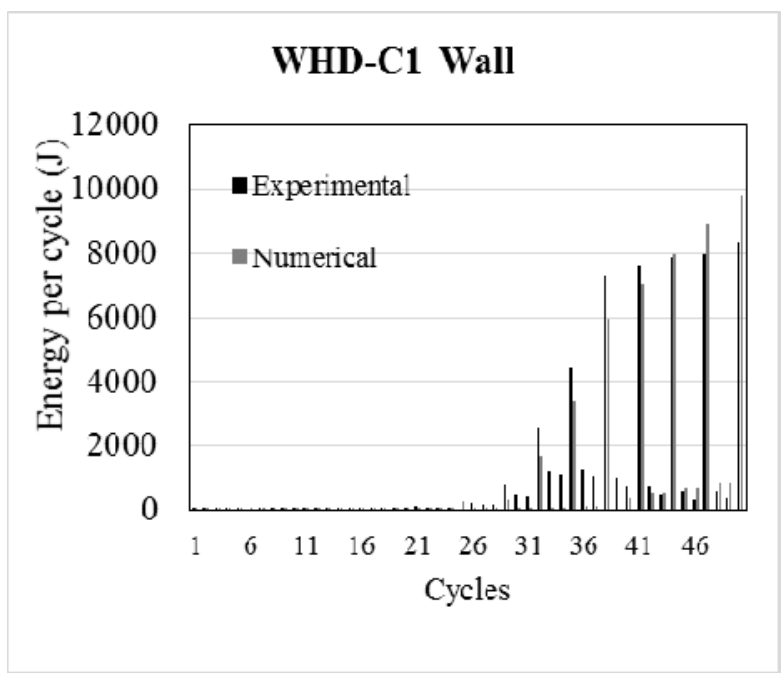

(b)

Figure 11. Comparison of a) Cumulative Energy Dissipation of Experimental and Numerical Results for the WHD-C1 Wall b) Cycle by Cycle Energy Dissipation of Experimental and Numerical Results for the WHD-C1 Wall

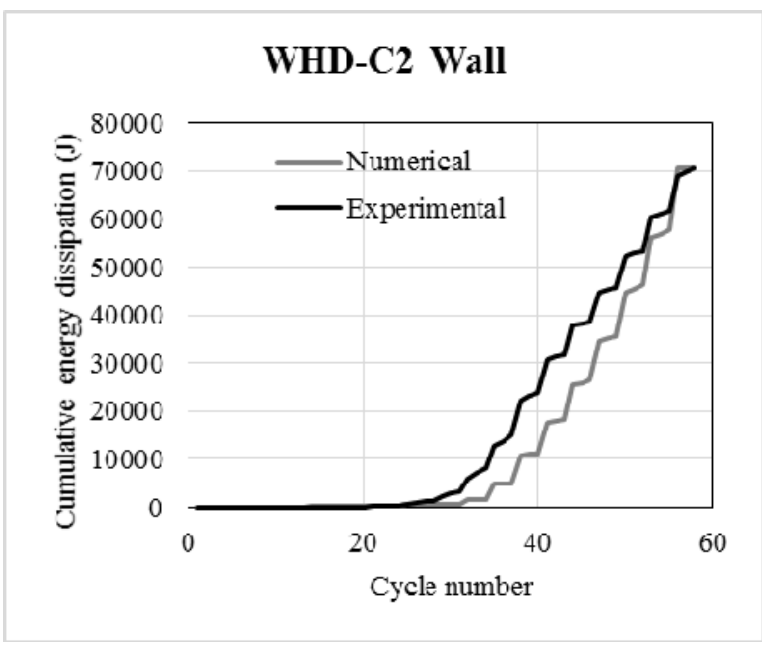

(a)

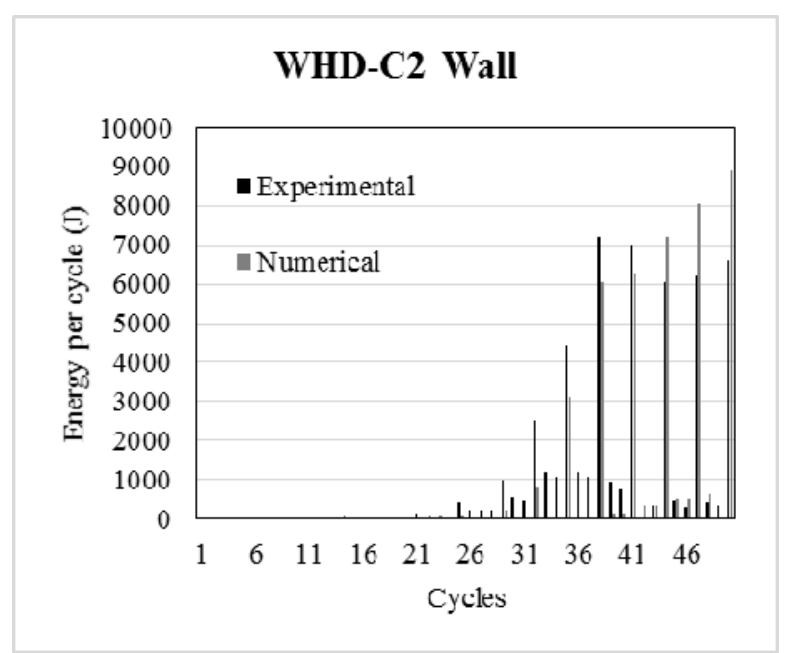

(b)

Figure 12. Comparison of a) Cumulative Energy Dissipation of Experimental and Numerical Results for the WHD-C2 Wall b) Cycle by Cycle Energy Dissipation of Experimental and

Numerical Results for the WHD-C2 Wall

As far as WHD-C1 and WHD-C2 walls are concerned, the comparison between cumulative and cycle by cycle energy dissipation for the numerical model and experimental results is shown in Figures 11 and 12, respectively. Energy dissipation in trailing cycles between 31 and 42 is underestimated by the numerical model. For these cycles, experiment results depicted an increase in the area of hysteretic loops, which is not properly captured by the numerical model. This difference can be seen in the plot of the cycle 42 in Figure 7. On the contrary, for trailing cycles after the $42^{\text {nd }}$, the experimental dissipated energy decreases, whereas the numerical one increases. The comparison of the dissipated energy in major primary cycles shows higher differences respect to the WLD configuration. Indeed, the average of the absolute values of $\Delta_{\mathrm{E}, \mathrm{i}}$ is $13.2 \%$ and 23.2 for WHD-C1 and WHD-C2 walls, respectively. In terms of cumulative energy, the average of the 
absolute values of $\Delta_{\mathrm{CE}, \mathrm{j}}$ calculated for cycles from 32 to $n$ is $29.2 \%$ and $34.6 \%$ for WHD-C1 and WHD-C2 walls, respectively. A small difference is observed for $\Delta \mathrm{CE}, \mathrm{n}$ at the $n^{\text {th }}$ cycle, which is less than $2 \%$ for both walls. Overall, it can be noted that energy dissipated in numerical models is in a quite good agreement with the experimental results.

\section{HYSTERETIC MODEL BASED ON THEORETICAL PREDICTION OF THE BACKBONE RESPONSE CURVE}

In this section, a comparison between the numerical models calibrated using a theoretical approach and experimental results is presented. Motivation behind this comparison is to check the possibility to use a theoretical prediction procedure for backbone curve of the pinching 4 material for walls, which would not have any experimental results available.

Table 3. Nominal and Design Values of Wall Lateral Strength Associated with each Wall Component

\begin{tabular}{|c|c|c|c|c|}
\hline \multirow{2}{*}{$\begin{array}{l}\text { Strength Associated } \\
\text { with each } \\
\text { component }\end{array}$} & \multicolumn{2}{|c|}{ WLD } & \multicolumn{2}{|c|}{ WHD } \\
\hline & $\begin{array}{c}\text { Nominal Resistance } \\
(\mathrm{KN})\end{array}$ & $\begin{array}{l}\text { Design Resistance } \\
(\mathrm{KN})\end{array}$ & $\begin{array}{c}\text { Characteristic } \\
\text { Resistance (KN) }\end{array}$ & $\begin{array}{c}\text { Design Resistance } \\
(\mathrm{KN})\end{array}$ \\
\hline Hc, $\mathbf{d}^{\mathrm{a}}$ & 42.84 & 42.84 & 85.68 & 85.68 \\
\hline Hc, $\mathbf{c}^{\mathrm{b}}$ & 92.59 & 74.07 & 183.50 & 146.80 \\
\hline Hc, $\mathbf{g}^{\mathrm{c}}$ & 137.67 & 110.13 & 216.61 & 173.29 \\
\hline Hc,s $s^{d}$ & 137.53 & 137.53 & 346.81 & 346.81 \\
\hline Hc, $\mathbf{d}^{\mathrm{e}}$ & 110.50 & 110.50 & 323.98 & 323.98 \\
\hline Hc, $\mathbf{a}^{\mathrm{f}}$ & 87.21 & 69.77 & 140.54 & 112.44 \\
\hline Hc ${ }^{g}$ & 42.84 & 42.84 & 85.68 & 85.68 \\
\hline
\end{tabular}

a. wall strength associated to the yielding of steel straps.

$b$. wall strength associated to the diagonal to strap connection

c. wall strength associated to the net failure of strap connections

$d$. wall strength associated to the buckling of studs

$e$. wall strength associated to the buckling of tracks

f. wall strength associated to the tension and shear anchors

g. minimum wall strength

The theoretical backbone curve of the wall is based on the prediction of only three structural response parameters: (1) wall strength; (2) wall stiffness; and (3) ultimate displacement. Therefore, an elastic perfectly plastic backbone (without hardening) curve having a limited plastic branch is adopted for the theoretical approach. Wall strength and stiffness are evaluated according to the procedure described in [5], by using experimental mechanical properties of materials and components obtained in [7]. Values of theoretical wall strength and stiffness for WLD and WHD wall configurations are given in Table 3 .

Figure 13 illustrates the comparison between theoretical and experimental backbone curves for WLD and WHD wall configurations. In particular, the first point of the theoretical backbone curve is positioned at the middle of the elastic branch defined by the theoretical predicted stiffness, whereas the second point is positioned at the end of elastic branch, defined by the theoretical predicted wall strength. The third point is defined by the theoretical predicted wall strength and a displacement corresponding to the $5 \%$ of the inter-story drift ratio. Finally, the fourth point defines the vertical drop of strength after the displacement corresponding to the $5 \%$ inter-story drift ratio. 
The parameters governing cyclic behaviour (uForce, rForce and $r$ Disp) are set equal to those give in Table 4, obtained by the calibration of the model on the basis of the experiment results. Based on theoretical backbone curves, numerical models of two walls are analysed again, and their performance is checked in terms of force-displacement response curves and cumulative energy dissipation. Numerical results based on theoretical backbone curve predictions is then been compared with available experimental results.

Table 4. Pinching04 Material properties for tested walls

\begin{tabular}{ccccc}
\hline Wall ID & WLD-C1 & WLD-C2 & WHD-C1 & WHD-C2 \\
\hline ePf1 (N/mm2) & & Parameters for backbone curve & & 90301 \\
\hline ePf2 (N/mm2) & 45150 & 45150 & 75250 & 180601 \\
\hline ePf3 (N/mm2) & 89548 & 90301 & 177591 & 186621 \\
\hline ePf4 (N/mm2) & 97826 & 99331 & 186621 & 7525 \\
\hline ePd1 (mm/mm) & 7525 & 7525 & 30100 & 0.00147 \\
\hline ePd2 (mm/mm) & 0.00092 & 0.00110 & 0.00074 & 0.00405 \\
\hline ePd3 (mm/mm) & 0.00276 & 0.00276 & 0.00368 & 0.03623 \\
\hline ePd4 (mm/mm) & 0.02851 & 0.02538 & 0.03679 & 0.03642 \\
\hline uForce & 0.02869 & 0.02557 & 0.03697 & 0.00 \\
\hline rDisp & & Parameters for cyclic behavior & & 0.70 \\
\hline rForce & 0.00 & 0.00 & 0.00 & 0.01 \\
\hline
\end{tabular}

From Figure 14, it can be noticed that numerical models obtained on the basis theoretical backbone curves are able to represent the cyclic behaviour of walls with good accuracy. Test results showed more strength as compared numerical model based on theoretical backbone curves. This difference can be attributed to the strain hardening of the material. In fact, experimental tests on WLD and WHD wall configurations showed a strength hardening of $8.5 \%$ and $4.0 \%$, respectively, which is not accounted for by the theoretical backbone curve. Therefore, the absence of strength hardening in backbone curves results in the underestimation of strength and energy dissipation.

Figure 15 illustrates the comparison of cumulative energy dissipation for WLD and WHD wall configurations. Numerical models based on the theoretical backbone curves of both wall generally underestimates the energy dissipation with respect to the experimental results. In particular, for WLD walls, the average of the absolute values of $\Delta \mathrm{E}, \mathrm{i}$ considering major primary cycles is $17.0 \%$ and $24.3 \%$ for WLD-C1 and WLD-C2 walls, respectively. Similar values are obtained for the mean of the absolute values of the cumulative energy error $\Delta_{\mathrm{CE}, \mathrm{j}}$ (from 32 to $n$ ), which are equal to $21.6 \%$ and $14.3 \%$ for WLD-C1 and WLD-C2, respectively. The value of $\Delta_{\mathrm{CE}, \mathrm{n}}$ at the $n^{\text {th }}$ cycle ranges between $12 \%$ and $15 \%$.

For the WHD wall configurations the comparison in terms of dissipated energy showed higher differences with respect to the WLD wall configurations. The average of the absolute values of $\Delta \mathrm{E}$, is $21.6 \%$ and $14.3 \%$ for WHD-C 1 and WHD-C2 walls, respectively. In terms of cumulative energy, the average of the absolute values of $\Delta_{\mathrm{CE}, \mathrm{j}}$ calculated for cycles from 32 to $\mathrm{n}$ is $28.5 \%$ and $31.8 \%$ for WHD-C1 and WHD-C2 walls, respectively. The value of $\Delta \mathrm{CE}, \Delta$ at the $n^{\text {th }}$ cycle is equal to $18.5 \%$ and 28.2 for WHD-C1 and WHD-C2 walls, respectively. The largest differences in terms of cumulative dissipated energy observed in the comparison with WHD-C2 wall are related to brittle rupture during the test, which cannot be accounted in OpenSees. Overall, the adoption of a theoretical predicted backbone curve seems to be reasonable for use in numerical models, at least for preliminary assessment of the hysteretic response. 

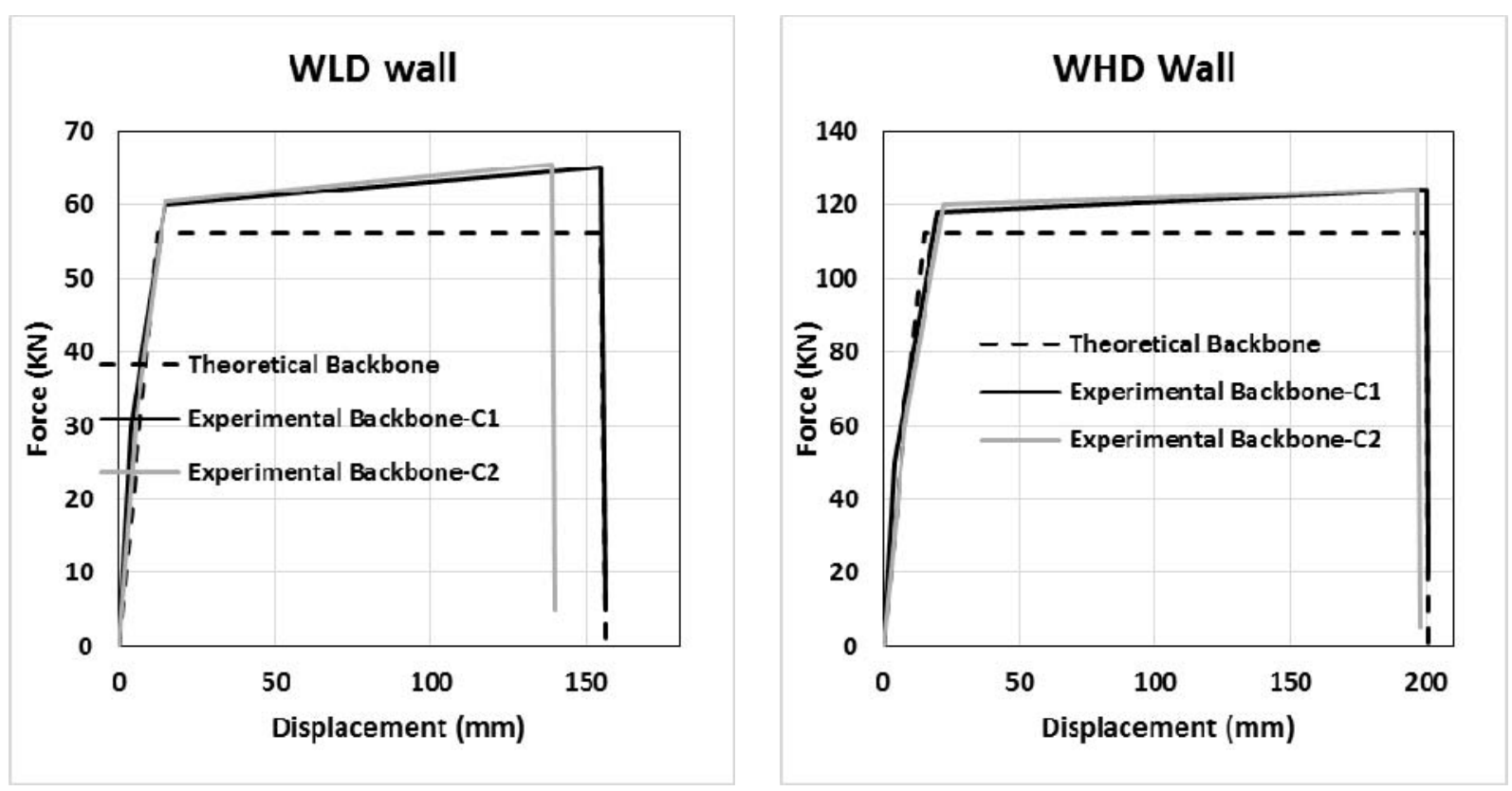

Figure 13. Comparison of Theoretical and Experimental Backbone Curve of WLD and WHD Wall Configurations

\section{BRITTLE FAILURE MODES IN NUMERICAL MODEL}

In order to show the ability of numerical models to capture brittle failure modes such as the tensile failure of tension anchors or buckling of chord studs, additional nonlinear cyclic analyses of walls with weak studs and anchors are performed. In fact, the walls modelled in this study are designed based on the capacity design approach, which ensures the ductile behaviour of system through proper over strength, it is not possible to observe any brittle failure modes in these walls. However, it is very important to ensure that the numerical models presented in this study are capable of simulating possible brittle failure modes for walls, which are not designed following the capacity design rules. In this context, two additional non-linear quasi static analyses are performed on WHD-C1 wall configuration with weak tension anchors and chord studs. Rest of the model parameters are same as that of experimentally calibrated WHD-C1 wall.

Anchors with $15 \%$ reduced tensile resistance $\mathrm{N}_{\mathrm{a}} \mathrm{Rk}$ corresponding to a wall strength $\mathrm{H}_{\mathrm{c} \text {,a }}$ of $140.5 \mathrm{kN}$ are used in a model with weak tension anchors. This resulted in tension anchor failing after the yielding of straps but prior to wall reaching its full ductility capacity. Failure is observed at a top wall displacement of $65 \mathrm{~mm}$ in comparison with $200 \mathrm{~mm}$ for wall designed based on capacity design rules. Figure 16 shows force vs displacement response curve of weak tension anchor model against the model designed based on capacity design rules.

Chord Studs with a $65 \%$ reduced buckling resistance $\mathrm{N}_{\mathrm{b}}$, Rk corresponding to a wall strength of $\mathrm{H}_{\mathrm{c}, \mathrm{s}}$ of $113.4 \mathrm{kN}$ are used in a weak stud model. This lead to buckling of chord studs after the yielding of straps and before the wall is able to reach its full ductility capacity. Failure occurred at a top wall displacement of $85 \mathrm{~mm}$ in comparison with $200 \mathrm{~mm}$ of wall designed following capacity design rules. Figure 17 shows the comparison of force vs displacement response curves of weak chord stud model against the model designed based on capacity design rules. 

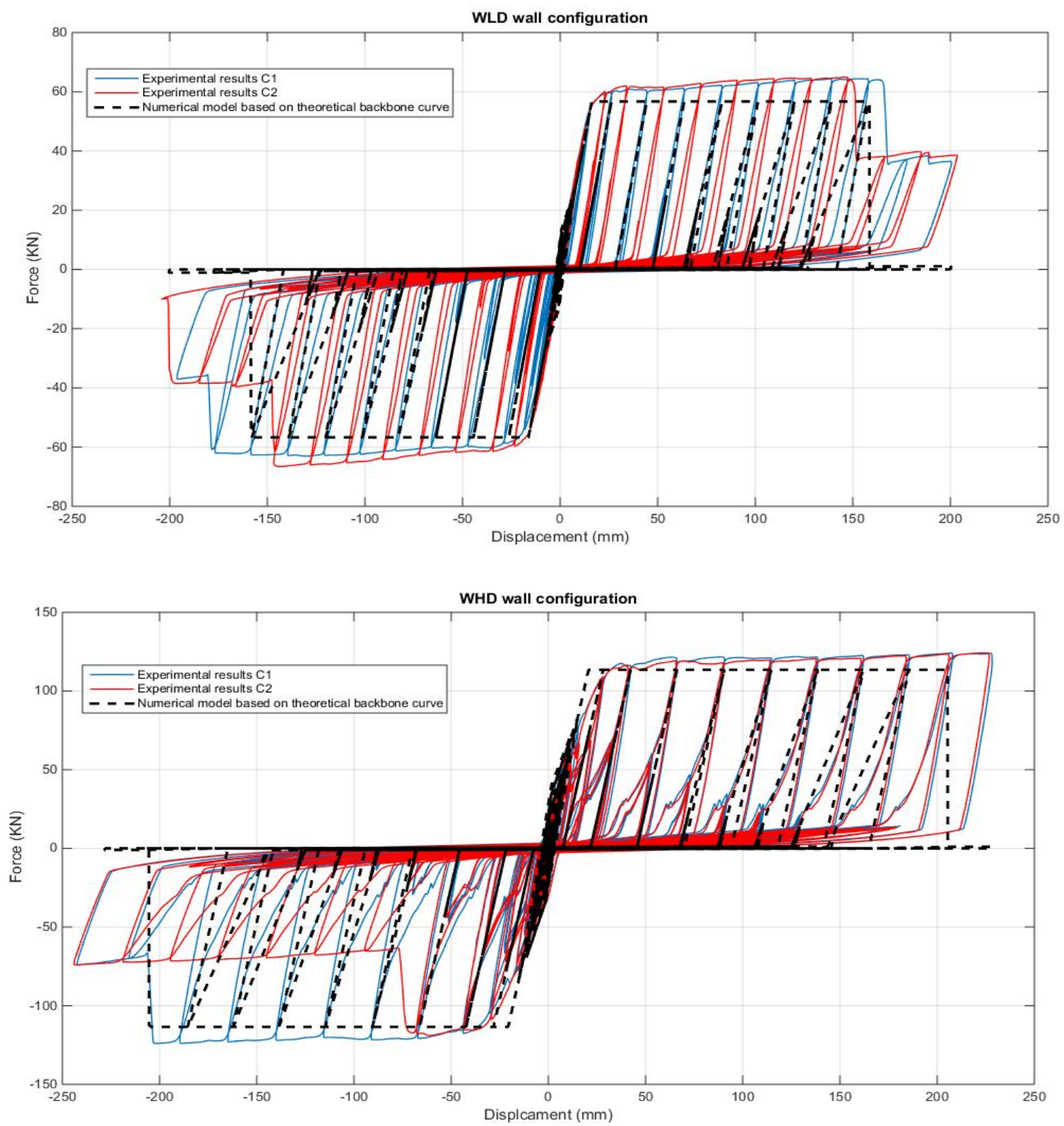

Figure 14. Comparison of Force vs. Displacement for WLD and WHD Walls 



Figure 35. Comparison of Cumulative Energy Dissipation of WLD and WHD Walls

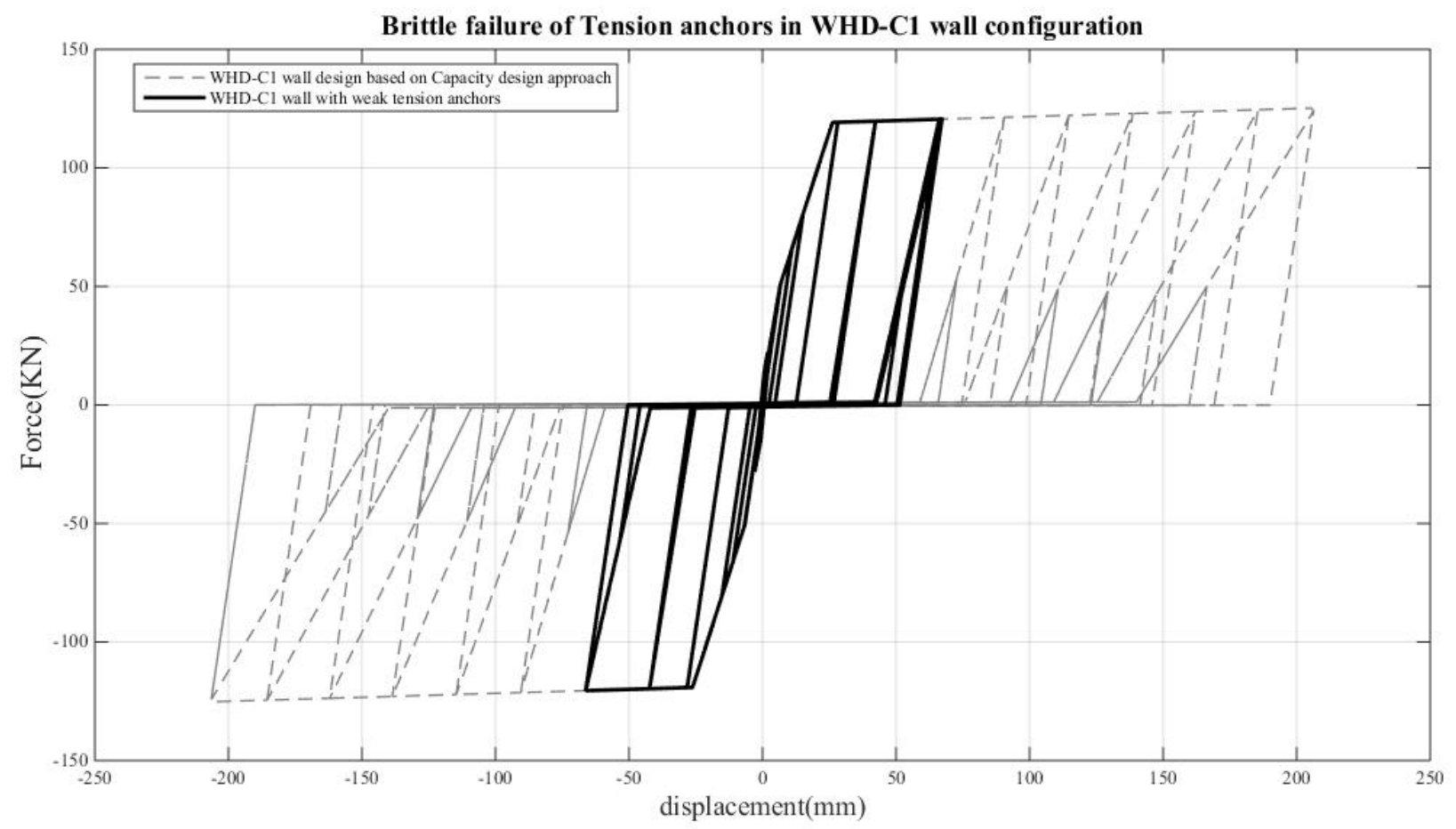

Figure 46. Force vs Displacement Response of Model with Weak Tension Anchors 


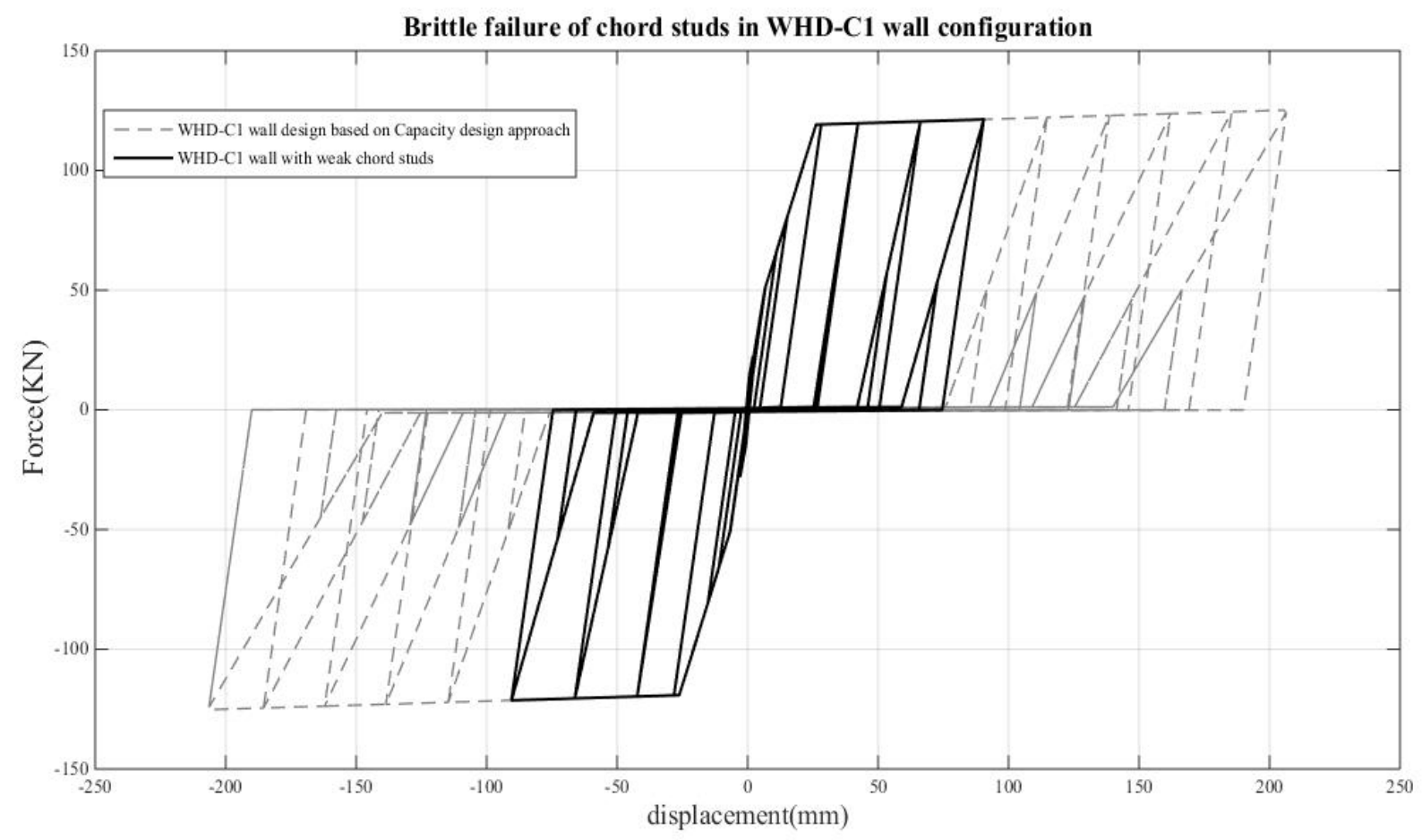

Figure 57. Force vs Displacement Response of Model with Weak Chord Studs

\section{CONCLUSIONS AND FURTHER DEVELOPMENTS}

In this paper development and calibration of numerical non-linear models in OpenSees using Pinching4 material for CFS strap braced stud walls is explained. Models are calibrated based on available experimental results. The developed model accurately captures the hysteretic behaviour of CFS strap braced stud walls. A good agreement in terms of hysteretic force-displacement response curves and energy dissipation is observed between numerical models and test results. Moreover, capability of numerical models based on the theoretical prediction of backbone curves to simulate hysteretic behaviour of CFS strap braced stud walls is explored, in order to provide evidence of their adequacy for walls, which do not have any experimental results available. Finally, the proposed numerical model shows the ability to capture also brittle failure mechanisms due to the collapse of chord studs and tension anchors, which represent possible behaviour in the case of inadequate design approach. In future, these models will be utilized to perform non-linear analysis on $3 \mathrm{D}$ buildings in order to evaluate the behaviour factor according to FEMA P695.

\section{ACKNOWLEDGEMENTS}

The authors acknowledge Italian Department of Civil Protection within RELUIS DPC 2014-2016 research project. This work has also been developed as thesis of European Erasmus Mundus master program on Sustainable Constructions under natural and catastrophic events (SUSCOS). Authors would also like to acknowledge European Commission (EACEA) for providing financial support. 


\section{REFERENCES}

[1] AISI-S400-15, "North American Standard for Seismic Design of Cold formed Steel Structural Systems," American Iron and Steel Institute (AISI), 2015.

[2] Fiorino, L., Iuorio, O., and Landolfo, R., "Seismic Analysis of Sheathing-braced Cold-formed Steel Structures", Engineering Structures, 2011, Vol. 34, pp. 538-547.

[3] Fiorino, L., Iuorio, O., MacIllo, V. and Landolfo, R., "Performance-based Design of Sheathed cfs Buildings in Seismic Area", Thin-Walled Structures, 2012, Vol. 61, pp. $248-257$.

[4] Iuorio, O., Fiorino, L. and Landolfo, R., "Designing cfs Structures: The New School bfs in Naples", Thin-Walled Structures, 2014, Vol. 78, pp. 37-47.

[5] Iuorio, O., Fiorino, L. and Landolfo, R., "Testing cfs Structures: The New School bfs in Naples", Thin-Walled Structures, 2014, Vol. 84, pp. 275-288.

[6] Macillo, V., Iuorio, O., Terracciano, M.T., Fiorino, L. and Landolfo, R., "Seismic Response of cfs Strap-braced Stud Walls: Theoretical Study", Thin Walled Structures, 2014, Vol. 85, pp. 301-312.

[7] Iuorio, O., Macillo, V., Terracciano, M.T., Pali, T., et al., "Seismic Response of cfs Strap-braced Stud Walls: Experimental Investigation", Thin-Walled Structures, 2014, Vol. 85, pp. 466-480.

[8] CEN, "EN 1998-1 Eurocode 8: Design of Structures for Earthquake Resistance-Part 1: General Rules, Seismic Actions and Rules for Buildings", European Committee for Standardization, Brussels, 2004.

[9] Fiorino, L., Iuorio, O., Macillo, V., Terracciano, M.T., et al., "Seismic Design Method for cfs Diagonal Strap-braced Stud Walls: Experimental Validation", Journal of Structural Engineering, 2015, No. 4015154.

[10] AISI-S213-07, "North American Standard for Cold-Formed Steel 2007 Edition with Supplement No.1", Vol. 9, American Iron and Steel Institute (AISI), Washigton, DC 2010.

[11] Mazzoni, S., McKenna, F., Scott, M.H., and Fenves, G.L., "Open System for Earthquake Engineering (Opensees)" n.d.

[12] Lowes, L.N., Mitra, N. and Altoontash, A., "A Beam-column Joint Model for Simulating the Earthquake Response of Reinforced Concrete Frames a Beam-column Joint Model for Simulating the Earthquake Response of Reinforced Concrete Frames," Berkeley 2004.

[13] Pastor, N. and Rodríguez-Ferran, A., "Hysteretic Modelling of x-braced Shear Walls", Thin-Walled Structures, 2005, Vol. 43, pp. 1567-1588.

[14] Kim, T., Wilcoski, J. and Foutch, D.A., "Analysis of Measured and Calculated Response of a Cold-formed Steel Shear Panel", Journal of Earthquake Engineering, 2007, Vol. 11, pp. $67-85$.

[15] Rakesh Allahabadi, G.H.P., "Drain-2dx”, 1988.

[16] Kim, T.-W., Wilcoski, J., Foutch, D.A. and Lee, M.S., "Shaketable Tests of a Cold-formed Steel Shear Panel”, Engineering Structures, 2006, Vol. 28, pp. 1462-1470.

[17] Comeau, G., Velchev, K. and Rogers, C.A., "Development of Seismic Force Modification Factors for Cold-formed Steel Strap Braced Walls", Canadian Journal of Civil Engineering, 2010, pp. 236-249.

[18] Federal Emergency Managment Agency (FEMA), "Quantification of Building Seismic Performance Factors", Washigton, DC, USA 2009.

[19] Carr, A.J., "Ruaumoko", 2000.

[20] Mirzaei, A., Sangree, R.H., Velchev, K., Comeau, G., et al., "Seismic Capacity-based Design of Narrow Strap-braced Cold-formed Steel Walls", Journal of Constructional Steel Research 2015, Vol. 115, pp. 81-91.

[21] Gad, E.F., Chandler, A.M., Duffield, C.F. and St, G., "Lateral Behavior of Plasterboard -clad Residential Steel Frames", Journal of Structural Engineering, 1999, pp. 125. 
[22] Swanson Analysis Systems Inc. (SASI), “Ansys” 1994.

[23] Barton, A.D., "Performance of Steel Framed Domestic Structures Subjected to Earthquake Loads", Faculty of Engineering, Civil and Environmental Engineering, University of Melbourne, 1997.

[24] Zeynalian, M. and Ronagh, H.R., "Thin-walled Structures a Bumerical Study on Seismic Performance of Strap-braced Cold-formed Steel Shear Walls", Thin Walled Structures, 2012, Vol. 60, pp. 229-238.

[25] Shamim, I. and Rogers, C. a., "Steel Sheathed/cfs Framed Shear Walls under Dynamic Loading: Numerical Modelling and Calibration”, Thin-Walled Structures, 2013, Vol. 71, pp. $57-71$.

[26] Shamim, I. and Rogers, C.A., "Numerical Evaluation: aisi s400 Steel-sheathed cfs Framed Shear Wall Seismic Design Method", Thin-Walled Structures, 2015, Vol. 95, pp. 48-59.

[27] Leng, J., Schafer, B.W. and Buonopane, S.G., in:, Struct. Stab. Res. Counc. Annu. Stab. Conf. 2013, SSRC 2013, 2013, pp. 426-442.

[28] Liu, P., Peterman, K., Yu, C. and Schafer, B., in:, 2012.

[29] Kechidi, S. and Bourahla, N., "Deteriorating Hysteresis Model for Cold-formed Steel Shear Wall Panel based on its Physical and Mechanical Characteristics", Thin-Walled Structures, 2016, Vol. 98, No. 421-430.

[30] CEN, "EN1993-1-1 Eurocode 3: Design of Steel Structures -Part 1-1: General Rules and Rules for Buildings", European Committee for Standardization, Brussels 2005.

[31] CEN, "En 1993-1-3 Eurocode 3: Design of Steel Structures-part 1-3: General Rules-supplementary Rules for Cold-formed Members and Sheeting”, 2006, Vol. 3.

[32] Krawinkler, H., Francisco, P., Ibarra, L., Ayoub, A. and Medina, R., "CUREE Publication No. W-02 Development of a Testing Protocol for Woodframe Structures," 2001.

[33] Velchev, K., Comeau, G., Balh, N. and Rogers, C.A., "Evaluation of the aisi s213 Seismic Design Procedures through Testing of Strap Braced Cold-formed Steel Walls", Thin-Walled Structures, 2010, Vol. 48, pp. 846-856. 